\title{
ANESTHESIA IN THORACIC SURGERY.
}

\author{
By C. LANGTON HEWER, M.B., B.S. (Lond.).
}

(Ancesthetist, St. Bartholomew's Hospital, Hospital for Tropical Diseases, Brompton Hospital and Seamen's Hospital, Royal Albert Dock.)

In thoracic surgery the success or otherwise of an operation frequently depends upon the successful administration of the anæsthetic, so that a consideration of this subject is of importance.

\section{Premedication.}

It need hardly be said that the patient should be got into the best possible condition before the operation, and for the I2 hours immediately preceding should have an adequate supply of fluids and of glucose.

Premedication must now be considered. It is desirable that the patient should be drowsy before the operation, but full basal narcosis is not always to be recommended, since it is important that the coughing reflex should have returned immediately after the operation, and, indeed, the patient should as a rule be able to cough voluntarily before leaving the table. If local analgesia only is contemplated, full basal narcosis is definitely unwise since reflex movements might occur and as the patient is non-cooperative he may become uncontrollable. For these reasons, it is the writer's practice to give a preliminary hypnotic such as morphine (or omnopon or opoidine) and scopolamine about $\frac{3}{4}$ hour before the operation. These drugs are usefully combined with atropin in the "Hyoscine Compounds 'A' and ' $B$ ' ", the former being suitable for the average female and the latter for a male. If a patient is known to react badly to morphia, two or three capsules ( 3 to $4 \frac{1}{2}$ grains) of nembutal are substituted. In children, rectal paraldehyde in doses of about 50 minims per stone body weight in a Io per cent. solution in water is useful. If for any reason a full basal narcosis is considered essential, intravenous sodium evipan should be used, as its rapid elimination usually leaves the patient with intact reflexes after operation.

Since the anæsthetic problems in thoracic surgery vary greatly with the type of proposed operation, it will be necessary to discuss them separately.

\section{Bronchoscopy and Esophagoscopy.}

In adults bronchoscopy and œsophagoscopy can usually be carried out under local analgesia if desired. The patient is given an adequate dose of morphine and scopolamine $\frac{3}{4}$ hour before the operation. A solution of 60 minims each of Io per cent. cocaine and I in rooo adrenalin is mixed in a glass. container and applied by means of wool rolled firmly on to a curved applicator. The lips and dorsum of the tongue are first painted. The swab is then held below the arches of the faucial pillars on each side of the uvula. The swab must be kept firmly applied to one spot without any brushing action or retching will be induced. Having anæsthetized each pyriform sinus, a pause of 20 minutes should enable œsophagoscopy to be performed. 
For bronchoscopy, however, a swab is saturated with the solution and introduced into the larynx between the cords for 30 seconds so that a few drops may trickle down the trachea. The patient should be warned against swallowing or toxic effects may be produced. If it is desired to abolish the cough reflex altogether during the bronchoscopy (e.g. for the removal of a foreign body), Io to I5 minims of the solution already described may be introduced into the mouth of the bronchoscope and will then trickle down the tube into the bronchus under observation.

In children or in nervous adults general anesthesia is usually preferable, any desired method of induction being used, and anæsthesia maintained through the tube of the bronchoscope with nitrous oxide-oxygen, ether or chloroform. Intravenous sodium evipan is occasionally satisfactory but usually depresses the reflexes insufficiently unless an inhalation anæsthesia is superimposed.

\section{Phrenicotomy, Thoracoscopy and Artificial Pneumothorax.}

Comparatively minor operations such as phrenicotomy, thoracoscopy, and the induction of artificial pneumothorax can conveniently be performed under local infiltration analgesia, the solution used being .05 per cent. novocaine and I in 200,000 adrenalin. The same remarks apply to the drainage of acute pneumococcal empyema in adults.

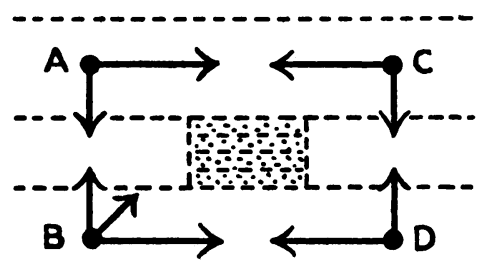

Fig. 1.-Local Analgesia for small rib resection.

For this purpose, four intradermal wheals are raised, A, B, C, and D, two in the intercostal space above and two in the space below the selected rib (Fig. I). An infiltration of the subcutaneous tissues and muscles joining the wheals is then made. Finally, from the lower posterior wheal B, a deep injection is made under the lower border of the selected rib in order to block the intercostal nerve in its groove. The needle must not be advanced deeper than $0.5 \mathrm{~cm}$. from the posterior surface of the rib, and the aspiration test must be negative before 2 c.c. of anæsthetic solution are injected. When the rib is exposed, a subperiosteal injection minimizes the pain of stripping the periosteum. The patient should always be warned when the pleura is about to be opened or the sudden sucking noise may cause great alarm.

Children are as a rule unsuitable for local analgesia and empyemata in them can be drained under gas and oxygen. It is also generally held that patients with streptococcal empyemata should be given a general anæsthetic, unless they 
are extremely ill, as extensive sloughing sometimes follows local infiltration, especially when preliminary exploration with needles has been performed. When the pleura is incised, the patient's condition must be closely watched as shock may occur. The effects of the pressure changes will vary with the extent of the empyema. Small collections of pus will not interfere appreciably with the normal rush of air into the open chest producing an artificial pneumothorax, the mediastinum being then displaced away from the affected side. Slight positive pressure applied with the anæsthetic gases will minimise this effect. In complete empyemata, however, the pus is sometimes under high pressure and if it escapes rapidly the surgeon should be requested to impede its exit so that the mediastinum will only move slowly over towards the affected side. It should be noted in thoracic surgery that it is always sudden displacement of the viscera which produces shock.

\section{Thoracoplasty.}

We now come to major operations upon the thoracic wall, of which thoracoplasty is the most common. Since these patients are generally suffering from phthisis, bronchiectasis, or chronic empyema, they are often in very poor general condition, and it is important not to add to their toxæmia. In the writer's opinion, pure nitrous oxide-oxygen is undoubtedly the anæsthetic of choice for these operations. The patient should be placed on the operating table in the position which the surgeon requires, and if he remains comfortable with no dyspnœa or cyanosis, anæsthesia may be induced. A fairly high percentage of oxygen is usually advisable since the patient is mainly relying upon one lung, and is lying on his sound side. The head should be kept low and turned to one side in order that any secretion may not be inhaled. If the sputum is very copious as in cases of bronchiectasis, the chest should be drained by posturing immediately before the operation. In extreme cases it may be necessary to resort to the bronchial suction technique described later. It will be found that a rubber harness (such as Clausen's face-piece retainer) materially helps to secure an air-tight fit between the mask and the patient's face. In "difficult" patients, the addition of a trace of chloroform usually ensures a smooth anæsthesia, but the smallest quantity of ether has an irritating effect and should be avoided. Divinyl Ether is without this action, but it is premature as yet to forecast its use in thoracic surgery.

The anæsthetist must observe the onset of shock (usually indicated by a rising pulse rate and a falling pulse pressure), and advise the surgeon if it becomes marked, since these operations can usually be done in several stages. Anti-shock measures include the administration of the highest possible oxygen percentage consistent with anæsthesia, a rectal injection of hot ro per cent. glucose in saline, the tight strapping of the chest immediately after the operation, and the injection of 5 units of insulin. There is no doubt that the use of the diathermic cautery diminishes shock as it shortens the operative time, minimizes capillary oozing and keeps the tissues warm. 
Local analgesia alone is rarely entirely successful in thorocaplasty and if it is combined with general anæsthesia, post-operative pain appears to be increased.

\section{Apicolysis.}

Apicolysis can be performed with the same methods of anæsthesia. Some anæsthetists prefer ethylene-oxygen to nitrous oxide-oxygen and it certainly possesses some advantages, the chief of which are the higher permissible percentage of oxygen and smoother anæsthesia. On the other hand, some patients have an unpleasant period of nausea following the operation. The gas is also explosive in certain percentages of oxygen and possesses an unpleasant smell. The latter need not incommode the occupants of the theatre if the closed circuit method of carbon dioxide absorption is employed, but even this technique cannot be regarded as safe if the diathermic cautery is employed since any explosion is liable to have disastrous results.

Although it is yet too early to speak with certainty, cyclopropane seems likely to be of great service in thoracic surgery, since mixtures containing no less than 80 per cent. oxygen provide adequate anæsthesia and the respiratory excursion is very small. At the present time the cost of the gas is so high that the carbondioxide absorption method must be employed. The only serious disadvantage of cyclopropane appears to be its explosive property when mixed with certain proportions of air and oxygen.

\section{Thoracotomy.}

We now pass to the consideration of extensive thoracotomies such as pulmonary lobectomy or the complete removal of the lung for bronchiectasis or new growth, the removal of substernal thyroid and other intra-thoracic tumours, the removal of intra-thoracic foreign bodies, the repair of diaphragmatic herniæ, and the excision of a part of the cesophagus for malignant disease. As a rule the effect of sudden pressure changes when the pleura is opened is minimized by a preliminary induction of artificial pneumothorax, but the anæsthetist must be prepared to apply positive pressure at any time. This can be effected by means of a simple gas-oxygen administration with the face-piece held tightly in position. Intermittent-flow machines are preferable to continuous-flow ones for this work, since the pressure can be readily adjusted without altering the percentage of gases in the mixture. It occasionally happens that both the pleural cavities are opened simultaneously and in this event the anæsthetist must immediately apply positive pressure or the patient will inevitably die of asphyxia in a short time.

The presence of blood or pus in the bronchial tree frequently complicates pulmonary lobectomy, and there are two methods of dealing with the situation. In the first place; intra-bronchial ancesthesia can be used. In this case the affected lung is cut off entirely so that no blood or pus can be aspirated into the sound side. This method has the additional advantage that the lung being operated upon is practically immobile. The technique was worked out by Waters and consists in using a long intubation tube fitted with a pneumatic rubber collar. The curved end of the tube is guided into the bronchus on the sound side and the collar 
should then lie at the bifurcation of the trachea. The average distance from the vocal cords to the bifurcation is II to I5 centimetres, and an advance of 2 to 3 centimetres beyond the bifurcation is sufficient. On inflating the collar with air from a syringe, the affected lung will be put out of action and to and fro respiration is carried on through the tube by the other lung with slight positive pressure (Fig. 2).

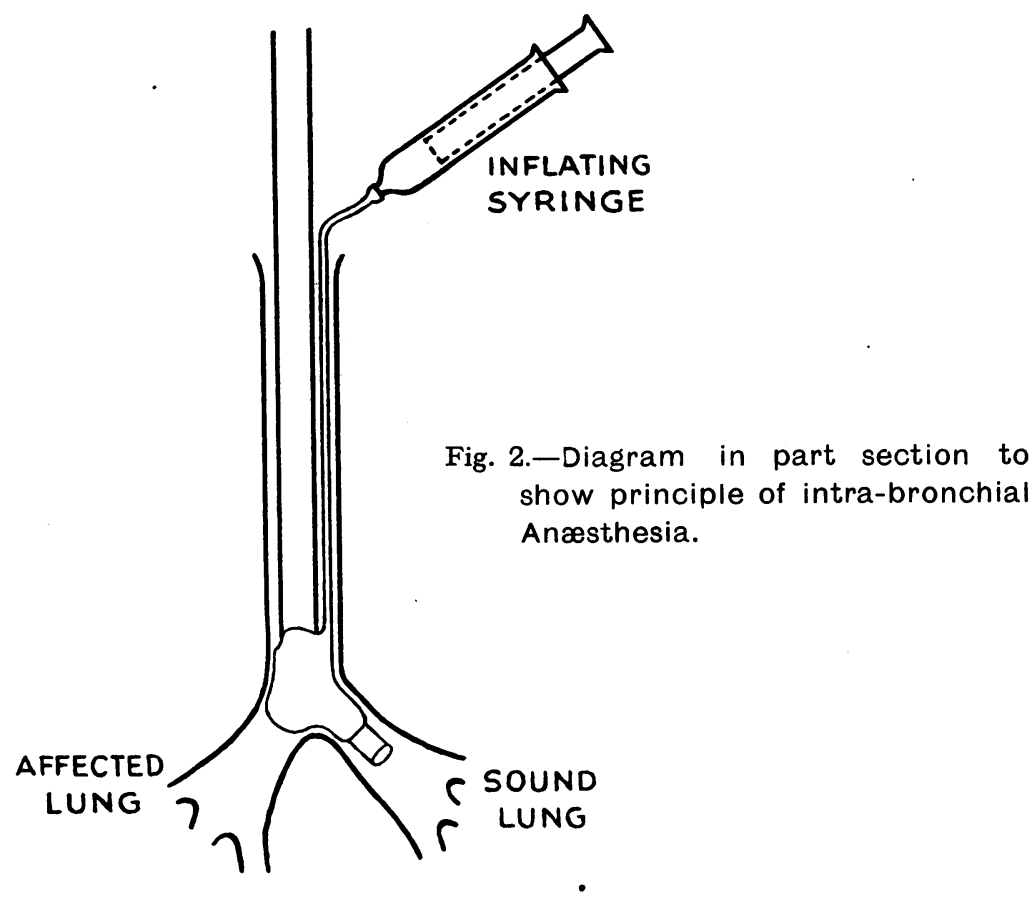

An alternative and easier method is endo-tracheal ancesthesia combined with bronchial suction, which was introduced by Magill. The usual soft rubber tube is introduced into the trachea through the nose and then a small stiff coudé catheter is passed down in front of this tube by direct laryngoscopy through the mouth. The end of the catheter should lie at the bifurcation of the trachea. A gauze pack is inserted around both tubes and nitrous oxide-oxygen anæsthesia maintained on the inhalation principally through the rubber tube. The orifice of the catheter is normally kept closed with a spigot to avoid an air leak, but may at any time be connected with a suction pump to clear the trachea and bronchi of fluid without disturbing the anæsthesia (Fig. 3). In the writer's experience this technique has proved very satisfactory.

Certain diseases for which thoracotomy is necessary may give rise to severe respiratory obstruction, of which the commonest is intra-thoracic goitre. In certain large tumours the embarrassment may be such that respiration is only possible with the head held forward, any attempt at extension of the neck pulling up the tumour which then compresses the trachea between the sternum in front and the vertebral column behind. In these cases, the maintenance of an efficient air-way outweighs every other consideration and the following technique may be adopted. 
If the goitre is producing toxic symptoms, preliminary basal narcosis with avertin may be desirable. The nose and glottis should then be cocainized and anæsthesia induced by means of nitrous oxide-oxygen, or possibly in extreme degrees of obstruction, by chloroform. A moderately rigid tube must then be inserted into the trachea either "blindly" through the nose or by direct laryngoscopy through

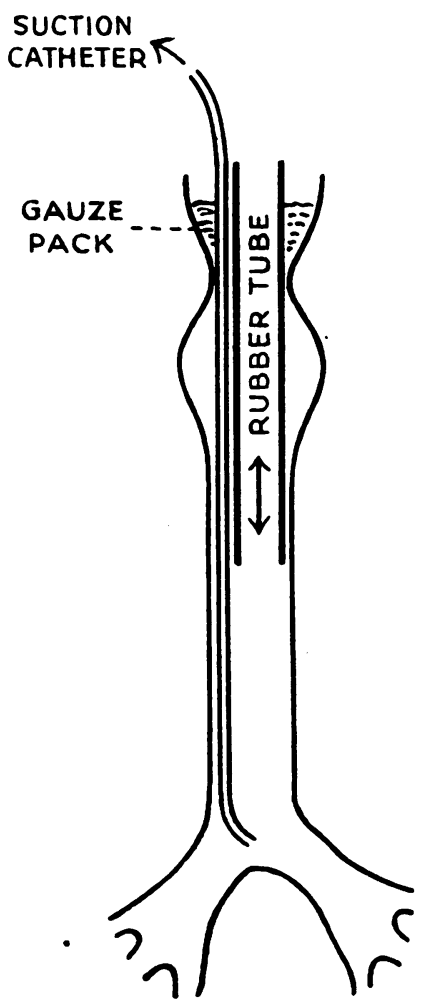

Fig. 3.-Diagram in section to show principle of endo-tracheal anæsthesia combined with bronchial suction.

the mouth. It is essential that the anæsthetist should be expert at intubation as entire cessation of respiration may occur until the tube is pushed past the point of obstruction. This tube must be sufficiently rigid to remain patent during the preliminary stages of the operation and sufficiently wide to allow to and fro respiration to occur through it. If in an exceptional case it proves impossible to insert a tracheal tube, anæsthesia will have to be maintained with the head held in the position in which the least respiratory obstruction occurs until the sternum is split, after which the pressure is relieved and no further difficulty should arise. After the operation the tracheal tube should be removed cautiously, for, if the trachea has been softened it may be necessary to reintubate.

If during a thoracotomy the diaphragmatic movement is so excessive that it embarrasses the surgeon, the phrenic nerve in its thoracic course may be blocked with novocain, after which the diaphragm will be temporarily paralyzed. In all extensive thoracotomies the patient's condition must be closely watched and antishock measures instituted directly they are indicated. A deliberate pause in the operation may enable an exhausted patient to recover sufficiently for further procedures to be carried out. 